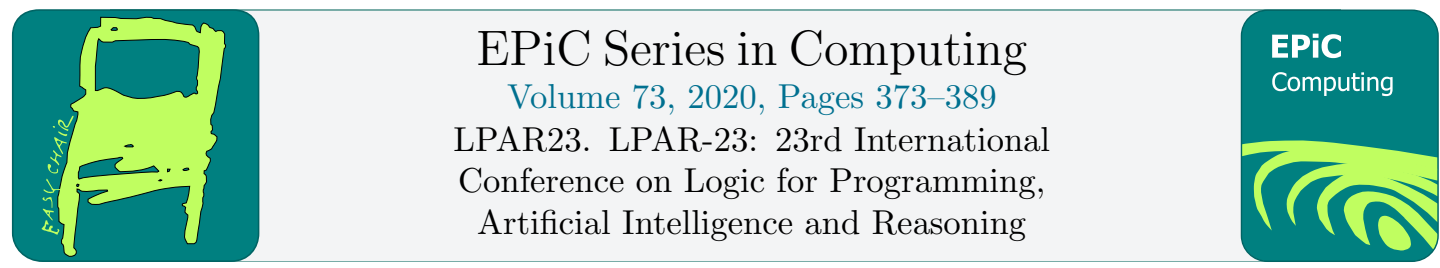

\title{
Coloring Unit-Distance Strips using SAT
}

\author{
Peter Oostema, Ruben Martins, and Marijn J. H. Heule \\ Carnegie Mellon University \\ Pittsburgh, Pennsylvania, United States \\ \{poostema, rubenm, mheule\}@andrew.cmu.edu
}

\begin{abstract}
Satisfiability (SAT) solving has become an important technology in computer-aided mathematics with various successes in number and graph theory. In this paper we apply SAT solvers to color infinitely long strips in the plane with a given height and number of colors. The coloring is constrained as follows: two points that are exactly unit distance apart must be colored differently. To finitize the problem, we tile the strips and all points on a tile have the same color. We evaluated our approach using two different tile shapes: squares and hexagons. The visualization of bounded height strips using 3 to 6 colors reveal patterns that are similar to the best known lower bounds for infinite strips. Our method can be a useful tool for mathematicians to search for patterns that can be generalized to infinite strips and allowed us to increase the lower bound for the strip height with 5 colors to an improved height of 1.700084 .
\end{abstract}

\section{Introduction}

The significant performance boost of SAT solvers in the last two decades have allowed them to efficiently deal with problems ranging from verification [4] to planning [13] and synthesis [11]. In this paper, we use these solvers for an application in computer-aided mathematics: coloring strips in the plane with a certain height under the unit-distance constraint. The unit-distance constraint states that two points must differ in color if their distance is exactly 1 (unit distance).

The goal of the presented work is to generate interesting patterns using a SAT solver by grouping neighboring points and give them the same color. A similar approach has been explored by Boris Alexeev, who showed that a square of 2.4 by 2.4 can be colored using six colors while satisfying the unit-distant constraint [1]. Mathematicians can study these patterns and determine whether they can be generalized.

Coloring unit-distance strips is related to the Chromatic Number of the Plane (CNP), a problem first proposed by Nelson in 1950 [18]. The CNP asks how many colors are required to color the entire plane using the unit-distance constraint. Early results showed that at least four and at most seven colors are required. By the de Bruijn-Erdős theorem, the chromatic number of the plane is the largest possible chromatic number of a finite unit-distance graph [8]. The Moser Spindle, a unit-distance graph with 7 vertices and 11 edges, shows the lower bound [14], while the upper bound is shown by a 7-coloring of the entire plane by Isbell [18]. Both the lower bound and the upper bound are shown in Figure 1. 


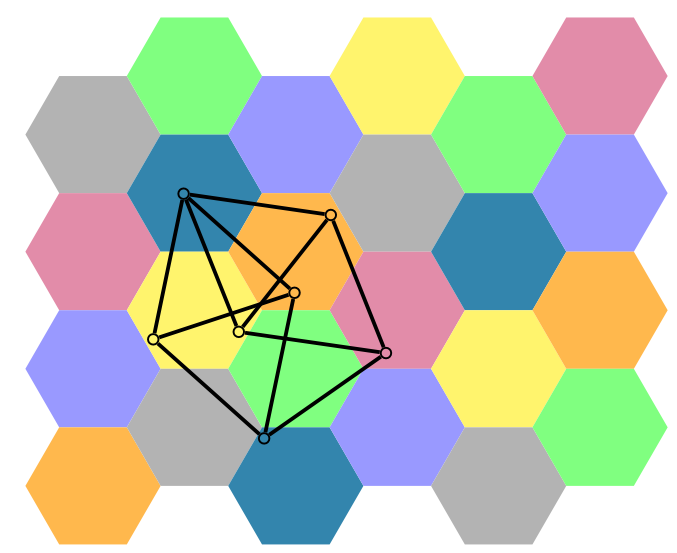

Figure 1: A 7-coloring of the plane using hexagon tiles with an outer radius of slightly smaller than unit distance. Also shown is a visualization of the Moser spindle graph.

In a breakthrough for the CNP in April 2018, Aubrey de Grey improved the lower bound by providing a unit-distance graph with 1581 vertices with chromatic number 5 [9]. This discovery by de Grey started a Polymath project to find smaller graphs. Various smaller graphs have been constructed with the help of SAT solvers [12]. The current record is a graph with 509 vertices and 2442 edges, which was recently discovered by Jaan Parts [16] and shown in Figure 2.

While these results provide understanding in lower bounds of the CNP, our research explores whether SAT solvers can be used to produce and help with upper bound results. Existing upper bound advances are based on a tessellation of the plane with shapes such that all points in the shape have the same color and all points in two shapes with the same color are at least distance 1 apart. Pritikin showed that there exists a 7-color tiling of the plane such that only a tiny fraction of the plane is colored with the seventh color [17].

In this paper, we focus on coloring infinite strips with a given height instead of the entire plane. Table 1 summarizes the research in this direction. Early results for 3 and 4 colors are based on coloring the strips with rectangles of the same shape and with a height that equals the strip height [2] and an example is shown in Figure 3a. A recent result improves the height for 4 colors using a much more involved pattern [6] and is shown in Figure 3b.

The only published result [2] for 5 colors is a height of 0.968 . A height of 1.625 has been

Table 1: Colorings of infinite strips with different heights

\begin{tabular}{c|l}
\hline \# Colors & Height \\
\hline 3 & $\sqrt{3} / 2 \simeq 0.866[3]$ \\
\hline \multirow{2}{*}{4} & $2 \sqrt{2} / 3 \simeq 0.94[3]$ \\
& $\sqrt{32 / 35} \simeq 0.956[15]$ \\
& $0.959[6]$ \\
\hline \multirow{2}{*}{5} & $\sqrt{15} / 4 \simeq 0.968[2]$ \\
& $13 / 8 \simeq 1.625[15]$ \\
& $9 / 2 \sqrt{7} \simeq 1.70084[$ this paper] \\
\hline 6 & $\sqrt{15} / 2+\sqrt{3} \simeq 3.668[2]$ \\
\hline
\end{tabular}




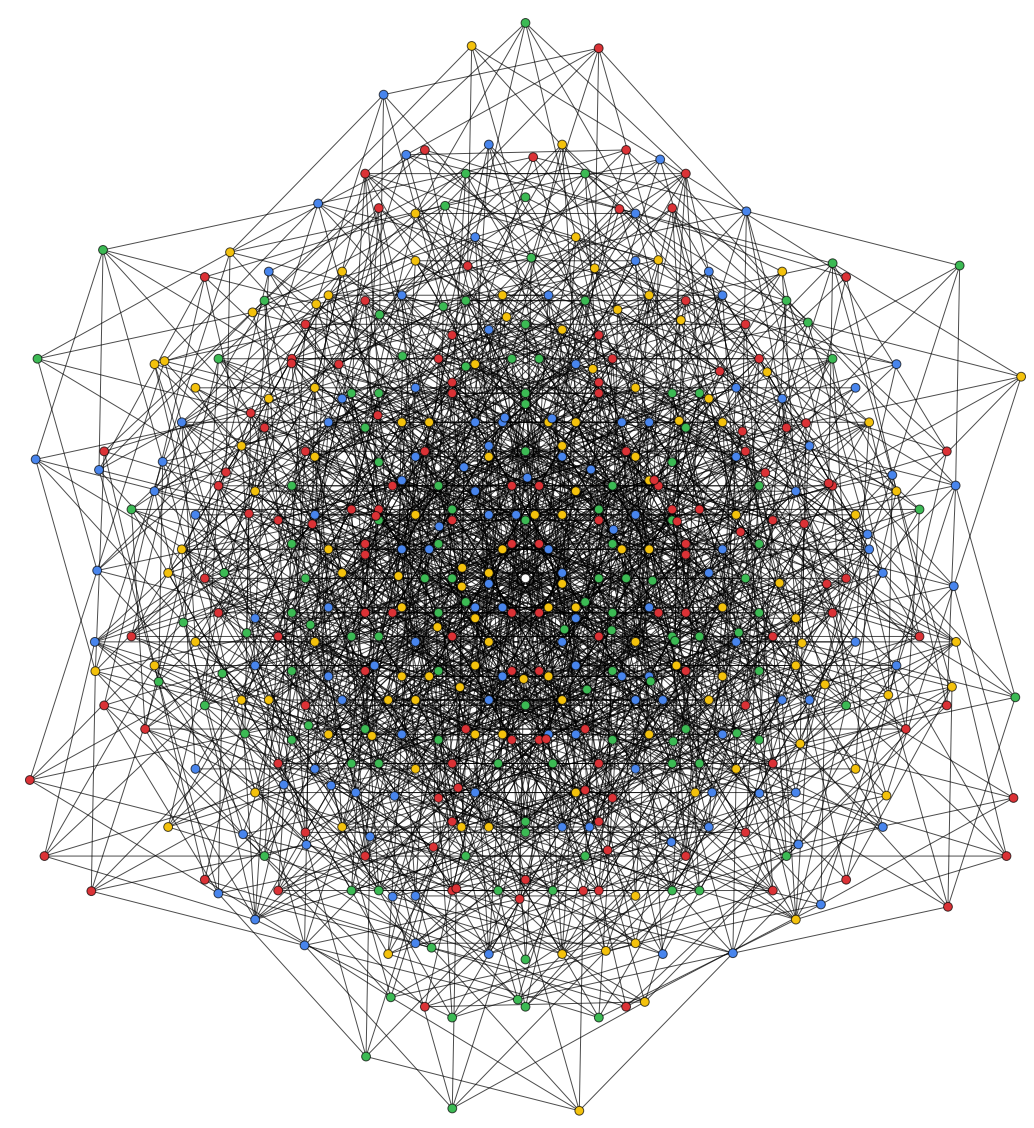

Figure 2: A unit-distance graph with chromatic number five with 509 vertices and 2, 442 edges. The vertices are colored with five colors. Only the central vertex has the fifth color (white), which results in patterns in for colors of the other nodes.

posted on a blog by Jaan Parts [15] showing a construction with pentagons. Our SAT-based approach also produces a coloring with similar shapes, but with a larger height. We constructed a tiling with height 1.70084 based on the SAT solution. The best known result for 6 colors is a height of 3.668 [2] and is shown in Figure 3c. Our SAT-based approach was able to find a similar pattern for a similar height after relaxing the constraints, which we call scaling.

\section{Background}

Below we present some background concepts related to the contributions of this paper.

\subsection{Chromatic Number of the Plane}

The Chromatic Number of the Plane (CNP) [18] asks how many colors are required in a coloring of the plane to ensure that there exists no monochromatic pair of points with distance 1. A unit-distance graph is a graph formed from a set of points in the plane by connecting two points 


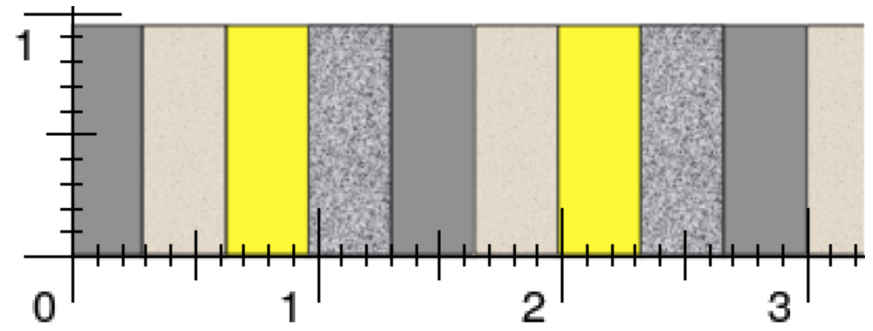

(a) 4-coloring of a strip with height 0.94 [2]

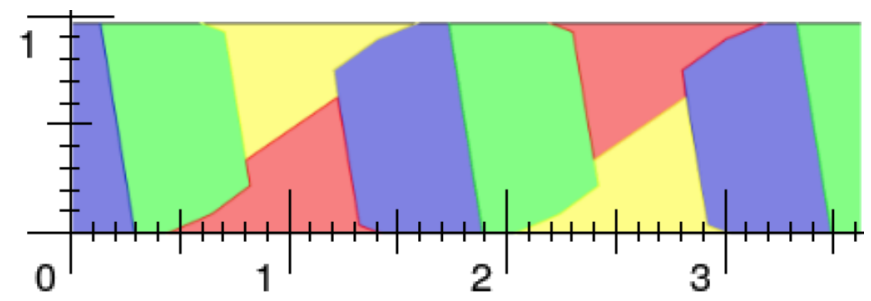

(b) 4-coloring of a strip with height 0.959 [6]

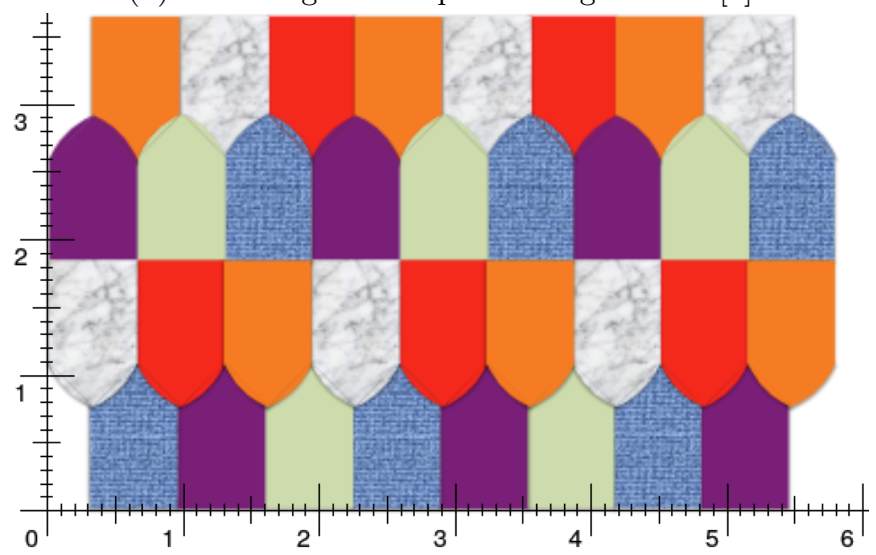

(c) 6-coloring of a strip with height 3.668 [2]

Figure 3: Some known $k$-colorings of strips

by an edge whenever the distance between the two points is exactly one. A lower bound for CNP of $k$ colors can be obtained by showing that a unit-distance graph has chromatic number $k$. An upper bound for CNP of $k$ colors can be obtained by showing that a $k$-coloring of the plane exists, such as the tessellation shown in Figure 1. In this paper, we will focus on coloring infinitely long strips of a given height.

\subsection{Graph Coloring}

Given a graph $G=(V, E)$ and a number $k$, the graph coloring problem asks whether it is possible to color the vertices $v \in V$ with $k$ colors such that for all edges $(v, w) \in E$ hold that vertices $v$ and $w$ have a different color. The chromatic number of a graph $G$, denoted by $\chi(G)$, is the smallest number $k$ such that there exists a valid $k$ coloring of $G$. To determine $\chi(G)$, one 
needs to show that a valid $\chi(G)$-coloring exists for $G$, while there exists no such coloring with $\chi(G)-1$ colors.

\subsection{Propositional Satisfiability}

We compute the chromatic number of graphs on the propositional level. We consider formulas in conjunctive normal form (CNF), which are defined as follows. A literal is either a variable $x$ (a positive literal) or the negation $\neg x$ of a variable $x$ (a negative literal). The complement $\neg l$ of a literal $l$ is defined as $\neg l=\neg x$ if $l=x$ and $\neg l=x$ if $l=\neg x$. For a literal $l, \operatorname{var}(l)$ denotes the variable of $l$. A clause is a disjunction of literals and a formula is a conjunction of clauses.

An assignment is a function from a set of variables to the truth values 1 (true) and 0 (false). A literal $l$ is satisfied by an assignment $\alpha$ if $l$ is positive and $\alpha(\operatorname{var}(l))=1$ or if it is negative and $\alpha(\operatorname{var}(l))=0$. A literal is falsified by an assignment if its complement is satisfied by the assignment. A clause is satisfied by an assignment $\alpha$ if it contains a literal that is satisfied by $\alpha$. A formula is satisfied by an assignment $\alpha$ if all its clauses are satisfied by $\alpha$. A formula is satisfiable if there exists an assignment that satisfies it and unsatisfiable otherwise.

\section{Coloring of Strips as a SAT problem}

To translate our problem into SAT we first need to create a tessellation of the strip and make a graph from the shapes that contain points one distance away. Several polygons can be used to tessellate the plane. In this work, we tessellated the plane with squares and hexagons. Squares have the advantage of being able to draw straight lines, while hexagons are one of the roundest shapes which minimizes the number of conflicts between intersecting shapes.

Each shape was given two indices (one for each dimension) to identify its position as depicted in Figure $4 \mathrm{a}$ for hexagons. We build a conflict graph where each shape is a node and there is an edge between two shapes if there is a conflict between them, i.e. they cannot be colored with the same color. The graph is constructed by finding the conflicts from one shape to all others and repeating that pattern for each shape. For instance, squares that touch the red outer border area in Figure 4b are conflicting with the central (black) square. These conflicts can be found efficiently by searching downwards for the first shape that is one distance away. From here the adjacent shapes are traversed to find the complete set of shapes that are one distance away. When a shape is found not in this range the traversal returns from that shape without adding a connection. The connections are stored at pairs of index offsets and easily translate to other shapes. For example, if two hexagons three rows apart share points one distance away then all hexagons will similarly share points with hexagons three rows above or below.

\subsection{SAT Encoding}

In this section, we show how to encode the coloring of strips to SAT. We follow a traditional encoding for graph coloring problems using SAT [10].

Variables. Let $S$ be the set of shapes and $K$ the set of colors. For each shape $s \in S$ we create $k=|K|$ variables $s_{1}, \ldots, s_{k}$. If $s_{i}$ is assigned truth value 1 then it means that shape $s$ is colored with color $i$. 


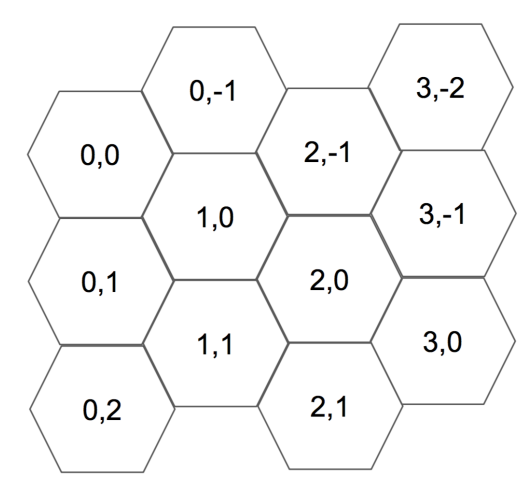

(a) Indexing of hexagons

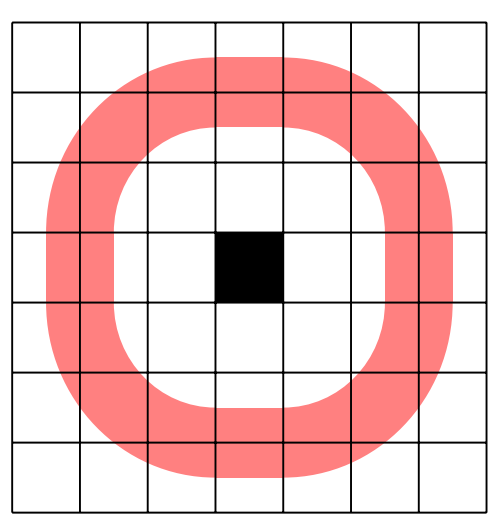

(b) Conflicts between squares

Figure 4: Indexing and conflicts in a tessellation

Constraints. There are two sets of constraints that we need to constrain our variables to encode a strip coloring problem. The first set of constraints requires that each shape $s \in S$ is colored by at least one of the $k$ colors. The following constraints capture this restriction:

$$
\bigwedge_{\forall s \in S \forall k \in K} \bigvee_{k}
$$

The second set of constraints uses the conflict graph constructed during the tessellation of the strip. Shapes that are connected with an edge cannot be colored with the same color. Let $E$ be the set of edges, where if $\left(s^{v}, s^{w}\right)$ is an edge in $E$ and $s^{v}, s^{w}$ correspond to the underlying shapes. The following constraints describe the conflict restrictions:

$$
\bigwedge_{\substack{\forall\left(s^{v}, s^{w}\right) \in E \\ \forall k \in K}}\left(\neg s_{k}^{v} \vee \neg s_{k}^{w}\right)
$$

Symmetry-breaking constraints. Like many other SAT problems, the coloring of strips has symmetries. To break some of the symmetries [7] that are associated with the permutation of colors, we find a triangle in the graph representation of the problem with vertices one distance away. Let $s, s^{\prime}$, and $s^{\prime \prime}$ be the underlying shapes that correspond to the three vertices of the triangle, then we add unit clauses such that each shape is assigned a different color (1 to 3 ):

$$
\left(s_{1}\right) \wedge\left(s_{2}^{\prime}\right) \wedge\left(s_{3}^{\prime \prime}\right)
$$

Scaling factor. In addition to the necessary constraints from the traditional SAT encoding for graph coloring problems [10], we introduce a new scaling factor for the shapes. The scaling factor is a ratio between 0 and 1 and describes what size of the internal shape will be colored. If the scaling factor is set to 1 , then the entire shape needs to be colored. Otherwise, a hexagon or square will be created at the center of the original shape, but with edge length scaled down. An example is given in Figure 5 which shows a coloring using a scaling factor of 0.74 . Notice the internal hexagons with white space between them since those areas do not need to be colored. This allows us to find patterns for the strip that may violate the conflict constraints and not fully color the strip, but still give us an intuition on how the pattern should look like. The 


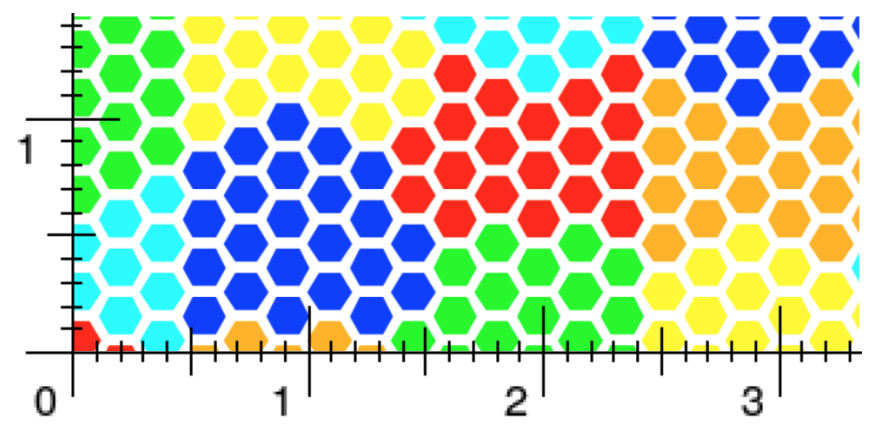

Figure 5: Coloring using a scaling factor of 0.74

scaling factor also has the added benefit of generating fewer conflicts between each shape and thus resulting in a smaller graph and easier to solve SAT formula. To improve the clarity of the images generated with a scaling factor, for the remainder of the paper, we will color the white space with the same color of the corresponding internal shape.

\section{Experimental Results}

To evaluate our approach, we encoded the $k$-coloring of a strip with variable height, fixed width of 8 , and $k \in\{3,4,5,6\}$. The tessellation of the plane was done with squares and hexagons. The experimental results presented in this section aim to answer the following research questions:

Q1: What is the largest height of a bounded strip that a SAT solver can find a $k$-coloring?

Q2: What is the impact of the different shapes in the coloring found by a SAT solver?

Q3: What is the impact of the scaling factor in the coloring found by a SAT solver?

The results described in this section were obtained using the Bridges system of the Pittsburgh Supercomputing Center. Nodes in the supercomputer have an Intel Xeon e5-2695 v3 @ $2.3 \mathrm{GHz}$ processor with 28 cores and $96 \mathrm{~GB}$ of memory. For this kind of problem, we observed that either the problem is solved within a short time limit or it does not get solved. Therefore, we run the SAT solver $\mathrm{CaDiCaL}^{1} 20$ times, using a different random seed each time, with a time limit of 600 seconds per run. Shuffling the formula or using random seeds to simulate a shuffling effect has been shown to increase the performance of SAT solvers to some classes of benchmarks [5], and we have observed similar phenomena while solving $k$-coloring strips problems. We implemented the encoding generator and reconstruction of the solution in our tool SArTcolor, which is written in Python and publicly available at GitHub. ${ }^{2}$

\section{$4.1 \quad k$-Coloring of Strips}

Table 2 shows the main results for $k$-coloring of strips using different colors and shapes without scaling. We can observe that SAT solvers can solve very large formulas, in particular, we were able to solve formulas with over 155 million clauses and 311 thousand variables. However, we can also observe that when the number of colors and the height of the strip increases, then

\footnotetext{
${ }^{1}$ https://github.com/arminbiere/cadical

${ }^{2}$ https://github.com/peteroostema/SATChromaticPlane
} 
Table 2: Largest strip height for $k$-colorings without scaling

\begin{tabular}{|c|c|c|c|c|c|c|c|c|c|c|}
\hline \multicolumn{2}{|c|}{ Strip } & \multicolumn{3}{|c|}{ Shape } & \multicolumn{4}{c|}{ Formula } & \multicolumn{4}{c|}{ Time (s) } \\
\hline \#Colors & Height & Polygon & Edge Length & Scaling & \#Vars & \#Clauses & \#Timeouts & Average & Std Dev & Result \\
\hline 3 & 0.86 & Square & 0.02 & 1 & 51,600 & $10,347,829$ & 0 & 3.20 & 0.042 & SAT \\
\hline 3 & 0.88 & Square & 0.02 & 1 & 52,800 & $10,939,217$ & 0 & 4.12 & 0.103 & UNSAT \\
\hline 3 & 0.82 & Hexagon & 0.01 & 1 & 77,841 & $1,7491,899$ & 0 & 6.50 & 0.0342 & SAT \\
\hline 3 & 0.84 & Hexagon & 0.01 & 1 & 80,250 & $18,740,099$ & 0 & 5.76 & 0.385 & UNSAT \\
\hline 4 & 0.94 & Square & 0.02 & 1 & 75,200 & $17,162,539$ & 0 & 6.52 & 0.034 & SAT \\
\hline 4 & 0.96 & Square & 0.02 & 1 & 76,800 & $18,109,011$ & 0 & 164.59 & 11.437 & UNSAT \\
\hline 4 & 0.92 & Hexagon & 0.01 & 1 & 116,628 & $30,468,376$ & 0 & 13.51 & 0.433 & SAT \\
\hline 4 & 0.94 & Hexagon & 0.01 & 1 & 118,768 & $31,841,591$ & 0 & 409.48 & 76.263 & UNSAT \\
\hline 5 & 1.66 & Square & 0.02 & 1 & 166,000 & $73,351,133$ & 18 & 400.84 & 24.148 & SAT \\
\hline 5 & 1.68 & Square & 0.02 & 1 & 168,000 & $74,808,263$ & 20 & - & - & Unknown \\
\hline 5 & 1.64 & Hexagon & 0.01 & 1 & 256,800 & $127,160,378$ & 19 & 407.75 & - & SAT \\
\hline 5 & 1.66 & Hexagon & 0.01 & 1 & 259,475 & $129,333,043$ & 20 & - & - & Unknown \\
\hline 6 & 1.80 & Square & 0.02 & 1 & 216,000 & $100,254,051$ & 19 & 419.29 & - & SAT \\
\hline 6 & 1.82 & Square & 0.02 & 1 & 218,400 & $102,002,527$ & 20 & - & - & Unknown \\
\hline 6 & 1.66 & Hexagon & 0.01 & 1 & 311,370 & $155,189,269$ & 4 & 229.90 & 7.303 & SAT \\
\hline 6 & 1.68 & Hexagon & 0.01 & 1 & 316,182 & $159,098,603$ & 20 & - & - & Unknown \\
\hline
\end{tabular}

the formulas become harder to solve. This also shows the importance of running the problem multiple times with different seeds since the SAT solver will perform a different search which may lead to a solution in a short amount of time. In general, using squares leads to finding colorings with larger heights than with hexagons. This may be explained by the fact that squares fit the space between the strip better than hexagons since at the strip border the hexagon will not draw a straight line and will have a small part dangling outside of the strip.

What is the largest height we can find a $k$-coloring without using a scaling factor? To answer this question, we fixed the edge length of hexagons to 0.01 and of squares to $0.02^{3}$ without using a scaling factor (i.e., scaling factor is set to 1) and increased the height of the strip in increments of 0.02 until it reached the best known value for that $k$-coloring or until we either got an unsatisfiable result from the SAT solver or 20 timeouts. Small squares and hexagons allow for more precise coloring and improve the quality of the image found by SArTcolor.

3-Coloring. Using squares, we were able to find a coloring for a strip of height 0.86 which is close to best known height for a 3-coloring of a strip (0.866). When increasing the height of the strip, the problem becomes unsatisfiable. In contrast, when using hexagons, we were only able to find a coloring for a strip with height 0.82 since the problem becomes unsatisfiable for larger heights. Figure 6 shows the coloring obtained by SArTcolor when using squares and hexagons for 3-coloring of strips. In both cases, we can see a repeating pattern. In Section 5, we will discuss how the repeating pattern in Figure 6a can be generalized to the infinite strip as described by Bauslaugh [3].

4-Coloring. For 4 colors, we found colorings of height 0.94 and 0.92 by using squares and hexagons, respectively. For larger height values, the SAT solver returned unsatisfiable. In this case, we did not found a 4-coloring for the largest known height of a strip which is 0.959 but our findings can still be helpful to aid mathematicians to generalize these colorings for infinite strips. Figure 7 shows the colorings returned by SArTcolor for the different shapes both

\footnotetext{
${ }^{3}$ Observe that the long diagonal of a hexagon with edge length of 0.01 is 0.02 .
} 


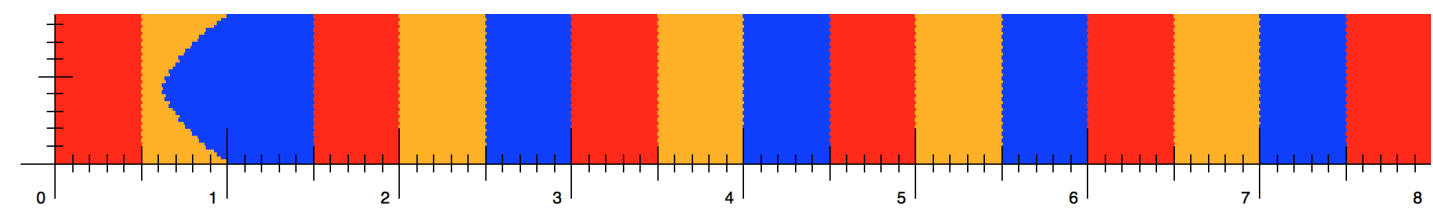

(a) Coloring using squares with edge length 0.02 and height 0.86

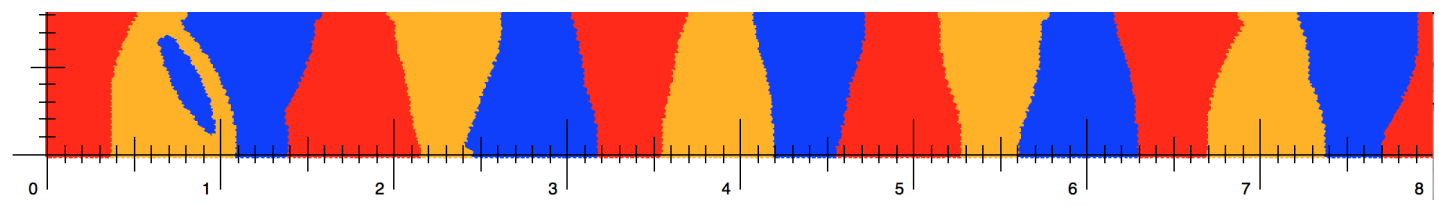

(b) Coloring using hexagons with edge length 0.01 and height 0.82

Figure 6: Comparison between shapes for 3-coloring of strips without scaling

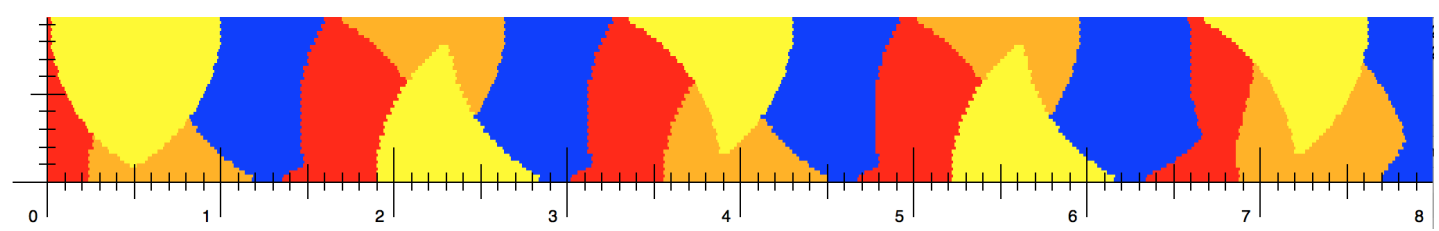

(a) Coloring using squares with edge length 0.02 and height 0.94

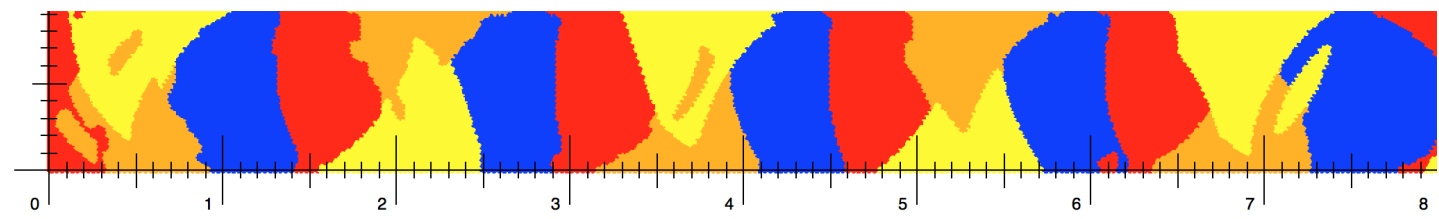

(b) Coloring using hexagons with edge length 0.01 and height 0.92

Figure 7: Comparison between shapes for 4-coloring of strips without scaling

revealing a similar pattern. This pattern resembles the best known 4-coloring [2] and will be further discussed in Section 5.

5-Coloring. For 5 colors, we found colorings with height 1.66 and 1.64 by using squares and hexagons, respectively. For larger height values, the SAT solver could not find any solution within the time limit. We can observe that some shapes have a coloring (e.g. red) that differs from the color of shapes in their close neighborhood (e.g. green). This is particularly visible in the upper left corner of Figure 8b. The reason for this noise is because some shapes can be colored with multiple colors while still leading to a valid coloring. As future work, we propose to perform a postprocessing step to smooth out this noise and get a more clear picture. The previously known best value for 5 colors was 1.625 [15] but in Section 5 we will show how the pattern depicted in Figure 8 can be generalized to an infinite strip with height 1.70084.

6-Coloring. The best known height for 6 colors is 3.668. However, we were only able to find a coloring for height 1.80 using squares and 1.66 using hexagons. The coloring problem 


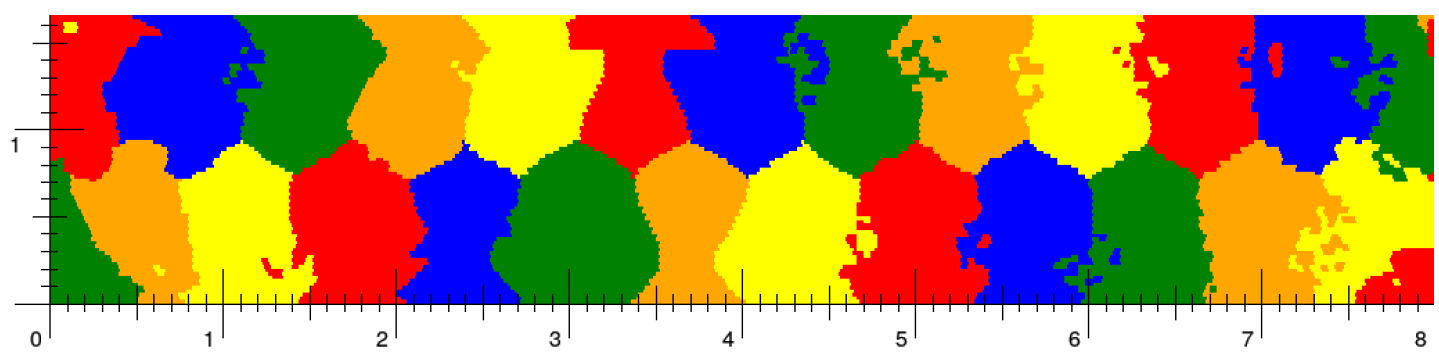

(a) Coloring using squares with edge length 0.02 and height 1.66

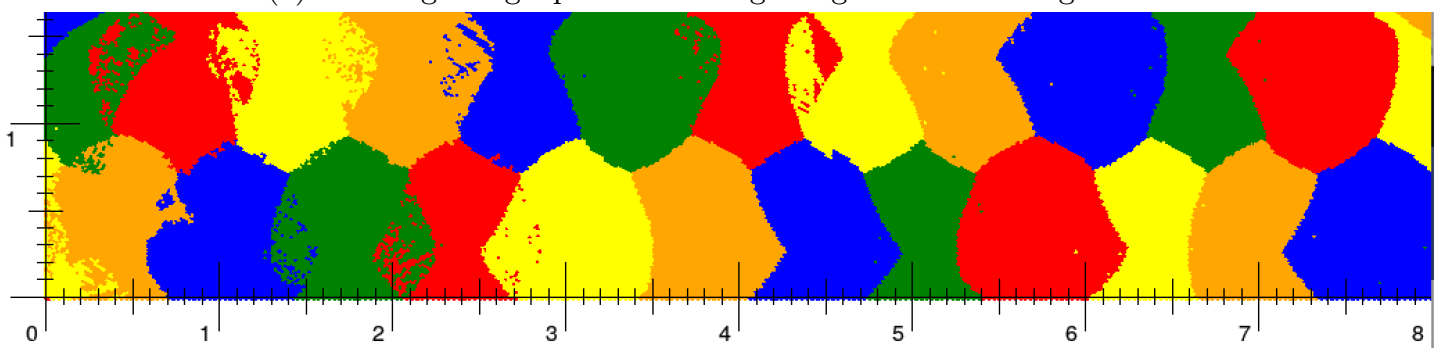

(b) Coloring using hexagons with edge length 0.01 and height 1.64

Figure 8: Comparison between shapes for 5-coloring of strips without scaling

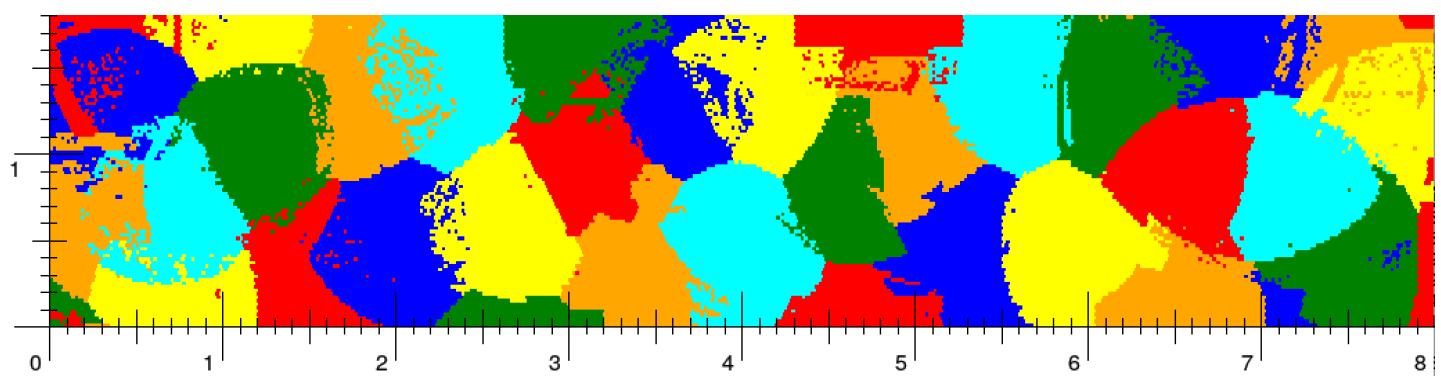

(a) Coloring using squares with edge length 0.02 and height 1.80

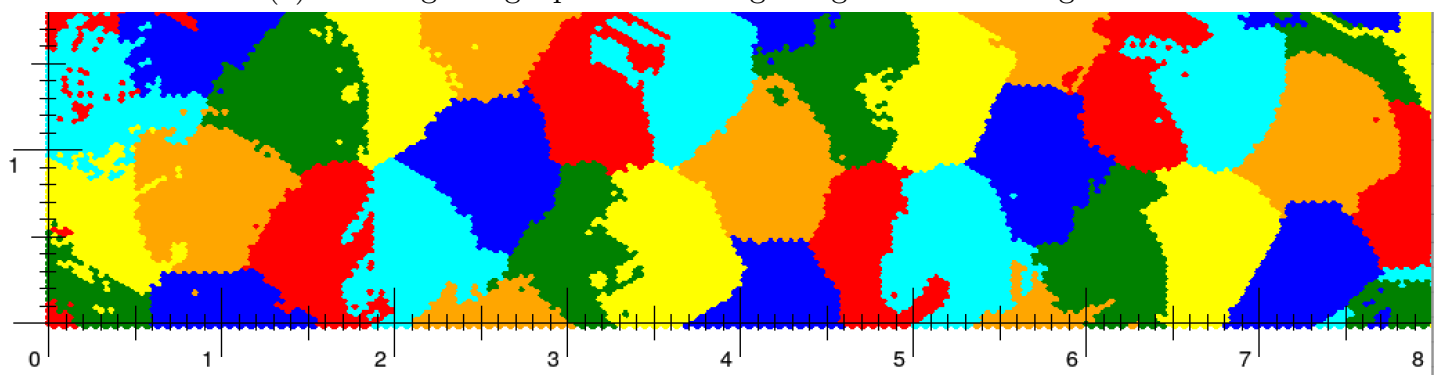

(b) Coloring using hexagons with edge length 0.01 and height 1.66

Figure 9: Comparison between shapes for 6-coloring of strips

becomes much harder to solve when the number of colors increases and as a consequence the gap between the best known height and the colorings found is large. 
Table 3: Configuration for $k$-colorings with heights fixed to the largest known values

\begin{tabular}{|c|c|c|c|c|c|c|c|c|c|c|}
\hline \multicolumn{2}{|c|}{ Strip } & \multicolumn{3}{|c|}{ Shape } & \multicolumn{4}{c|}{ Formula } & \multicolumn{4}{c|}{ Time (s) } \\
\hline \#Colors & Height & Polygon & Edge Length & Scaling & \#Vars & \#Clauses & \#Timeouts & Average & Std Dev & Result \\
\hline 3 & 0.86 & Hexagon & 0.01 & 0.2 & 81,855 & $3,182,553$ & 0 & 1.21 & 0.017 & SAT \\
\hline 3 & 0.86 & Hexagon & 0.01 & 0.3 & 81,855 & $6,534,258$ & 0 & 2.65 & 0.034 & UNSAT \\
\hline 4 & 0.96 & Square & 0.02 & 0.7 & 76,800 & $12,346,247$ & 0 & 4.16 & 0.128 & SAT \\
\hline 4 & 0.96 & Square & 0.02 & 0.8 & 76,800 & $14,239,867$ & 1 & 459.28 & 56.701 & UNSAT \\
\hline 4 & 0.96 & Hexagon & 0.02 & 0.5 & 31,088 & $2,139,723$ & 0 & 1.26 & 0.012 & SAT \\
\hline 4 & 0.96 & Hexagon & 0.02 & 0.6 & 31,088 & $2,580,555$ & 0 & 16.33 & 1.295 & UNSAT \\
\hline 5 & 1.70 & Square & 0.05 & 0.5 & 27,200 & $2,462,838$ & 4 & 230.22 & 194.48 & SAT \\
\hline 5 & 1.70 & Square & 0.05 & 0.6 & 27,200 & $3,069,148$ & 20 & - & - & Unknown \\
\hline 5 & 1.70 & Hexagon & 0.04 & 0.2 & 17,550 & 247,823 & 0 & 135.27 & 129.251 & SAT \\
\hline 5 & 1.70 & Hexagon & 0.04 & 0.3 & 17,550 & 621,273 & 20 & - & - & Unknown \\
\hline 6 & 3.66 & Square & 0.06 & 0.6 & 49,848 & $5,782,909$ & 19 & 264.85 & - & SAT \\
\hline 6 & 3.66 & Square & 0.06 & 0.7 & 49,848 & $7,445,413$ & 20 & - & - & Unknown \\
\hline 6 & 3.66 & Hexagon & 0.03 & 0.4 & 77,328 & $6,377,607$ & 18 & 289.14 & 297.119 & SAT \\
\hline 6 & 3.66 & Hexagon & 0.03 & 0.5 & 77,328 & $8,485,569$ & 20 & - & - & Unknown \\
\hline
\end{tabular}

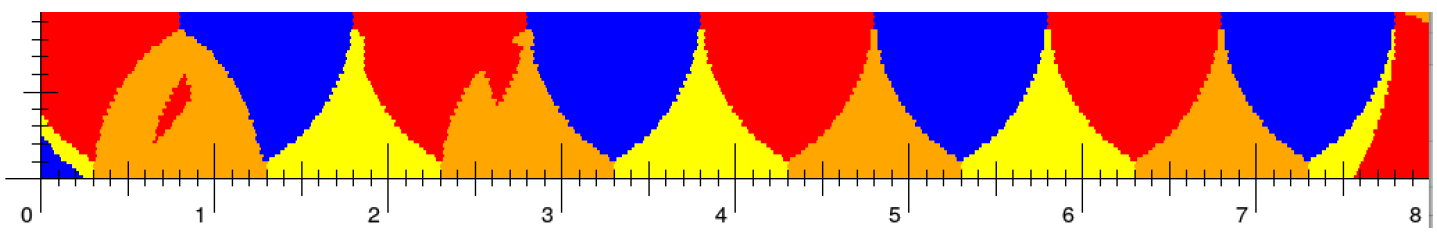

Figure 10: Coloring using squares with edge length 0.02 and scaling factor 0.7 for height 0.96

\subsection{Impact of Scaling Factor}

Table 2 shows that the largest height for which we can find $k$-coloring without scaling can be too far from the best known values, in particular for 6 colors. Table 3 shows the impact of the scaling factor when finding colorings close to the best known height values.

What is the scaling factor that we need to use to find a coloring for the heights of the best known $k$-colorings? We fixed the largest known height for each $k$-coloring problem and decreased the scaling factor until the SAT solver was able to find a solution. When doing this procedure, we were still not able to find any colorings by using an edge length of 0.02 for squares and 0.01 for hexagons. Therefore, in these cases, we also increased the edge length and found a coloring that maximized the scaling factor and minimized the edge length. Note that we did not perform this experiment for 3 colors using squares since we could already find a solution close to the largest known height. Even though we were always able to find a configuration for which the SAT solver found a solution, the quality of the coloring found depends on the scaling factor. For small scaling factors, the coloring can become too noisy to distinguish any pattern, which was the case for 5 colors using hexagons and a scaling factor of 0.2 . However, in most cases, using the scaling factor allowed us to see the same or new patterns at larger heights.

Figures 10 and 11 show the impact of scaling when using squares. We can observe a new coloring pattern in Figure 10 that was not observed in images without scaling. We will discuss a potential generalization of this pattern to an infinite strip in Section 5. For 6 colors, we observed a pattern similar to Figure $9 \mathrm{a}$ but at a larger height which makes the pattern more visible and easier to generalize to infinite strips. 


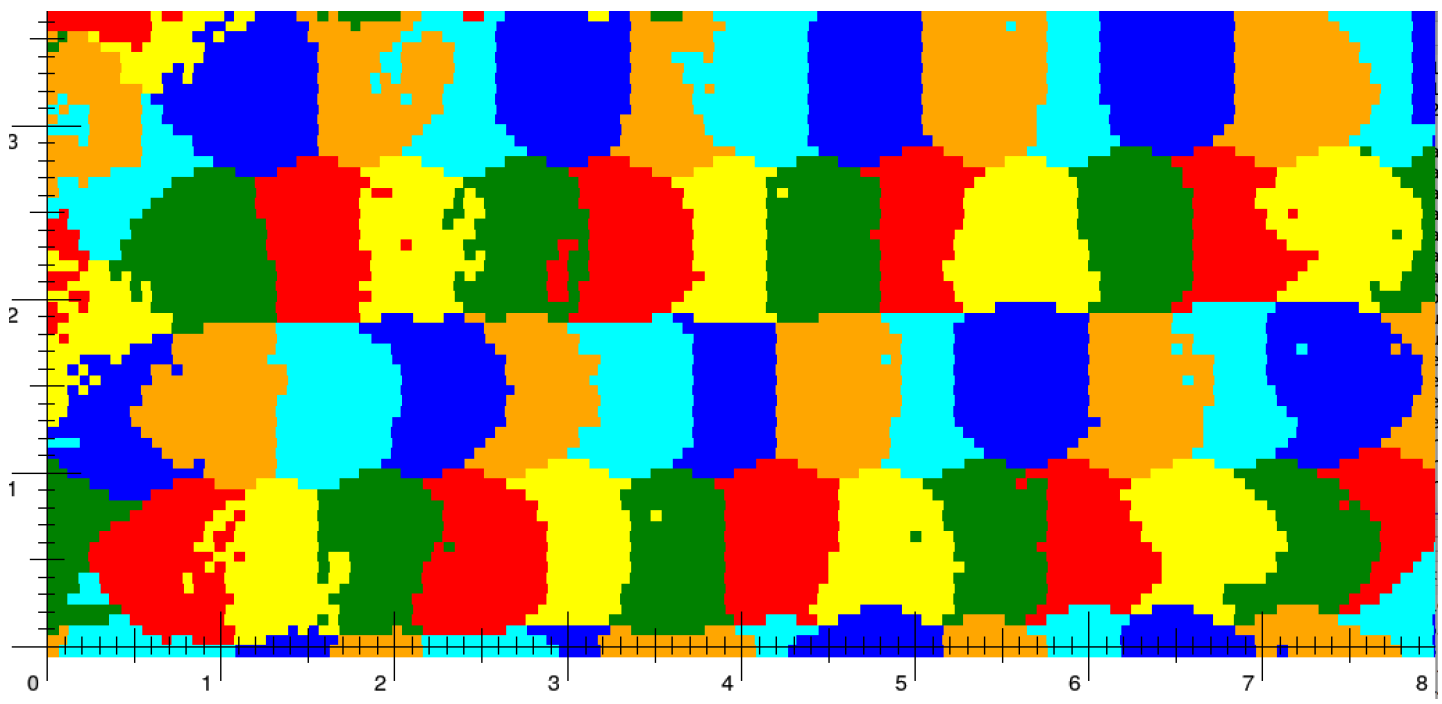

Figure 11: Coloring using squares with edge length 0.06 and scaling factor 0.6 for height 3.66

\section{Generalization to Infinite Strips}

In the previous section, we have shown that SAT solvers can find unit-distance colorings for bounded strips. To generalize the colorings found from bounded strips to infinite strips, we need to identify repeating patterns and check if these patterns can be used to obtain a valid coloring for infinite strips. In this section, we use the solution returned by the SAT solver as a starting point to find known generalizations for $k$-coloring of infinite strips and to find a new 5-coloring for infinite strips of height $\frac{9}{2 \sqrt{7}} \simeq 1.70084$.

\section{$5.1 \quad 3$-Coloring}

The pattern in Figure 6a can be used to construct a 3-coloring for an infinite strip with height $\frac{\sqrt{3}}{2} \simeq 0.866$ as shown by Bauslaugh [3]. This can be done by constructing an infinite strip of height $\omega=\frac{\sqrt{3}}{2}$ built of repeating monochromatic $\omega \times \frac{1}{2}$ rectangles with colors $\mathrm{R}$ (red), $\mathrm{O}$ (orange), B (blue), R, O, B, $\cdots$. Note that the same pattern can also be extended to the 4-coloring of a strip (see Figure 3a) with a height $\omega^{\prime}=2 \frac{\sqrt{2}}{3} \simeq 0.94$ built of monochromatic rectangles $\omega^{\prime} \times \frac{1}{3}[3]$ and to the 5-coloring of a strip with height $\omega^{\prime \prime}=\sqrt{\frac{15}{16}} \simeq 0.968$ and monochromatic rectangles $\omega^{\prime \prime} \times \frac{1}{4}[2]$.

Even though this is not a new result for 3-coloring, we can see that a SAT approach would give the exact pattern that generalizes to an infinite strip and can be used to provide insights to mathematicians of potential patterns that can be generalized.

\subsection{4-Coloring}

One pattern that emerged is shown in Figures $7 \mathrm{a}$ and $7 \mathrm{~b}$. These figures show two waves of alternating colors (yellow, orange) top and bottom separated by two colorings (blue, red) filling in the section between the waves. This is the same pattern as shown in Figure $3 \mathrm{~b}$ which resulted in the largest known height (0.959) for 4 colors. 


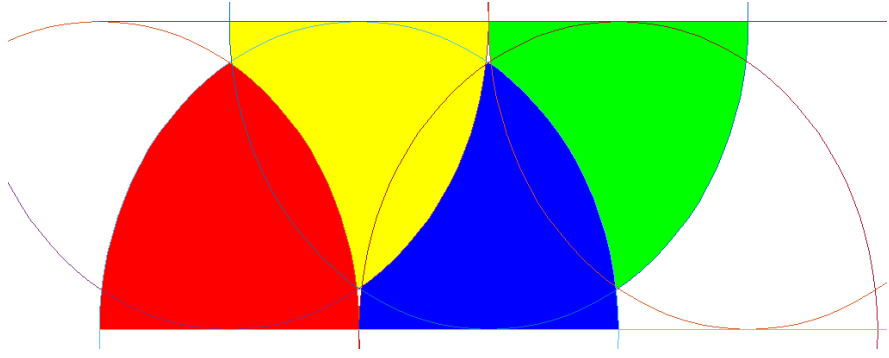

(a) An almost 4-coloring of a strip with height 1 pattern

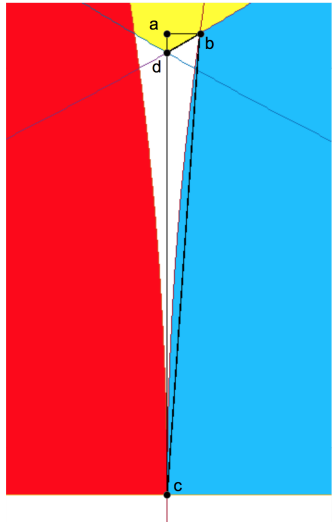

(b) Empty area of near 4-coloring

Figure 12: An almost 4-coloring of the strip with height 1

When using a scaling factor of 0.7, we observed a different pattern as shown in Figure 10. This has two colors repeated on the top (blue, red) and another two on the bottom (orange, yellow). We tried to generalize this pattern to an infinite strip of height 1. Figure 12a shows that this generalization cannot color a strip of height 1 completely since there is a small area in the intersection of the 3 colors that cannot be colored without breaking the unit-distance constraints. If we approximate the area that is not colored with two triangles and generalize it to the infinite strip, we can observe that this is an almost 4-coloring since we cover at least $99.74 \%$ of the strip. Consider the points $a, b, c$ and $d$ in Figure 12b. Notice that the endpoints of these triangles are the intersection of yellow, blue and white (point $b$ ), the intersection of red and blue at the bottom (point $c$ ), and the lowest point colored by yellow (point $d$ ). Point $a$ is the point in the middle and top making right triangles with these. First, the area is overapproximated with the right triangle with endpoints $a, b$, and $c$. Next, area is taken away from this triangle using another right triangle formed with the points $a, b$, and $d$. The coordinates of the points $a, b, c$, and $d$ are obtained using the geometric construction. The area that is not colored occurs four times in a unit distance square and that gives an approximation to the percentage of the colored area.

Even though our generalization was not successful, we can again see the usefulness of the SAT approach and the scaling factor by providing hints that can be used by mathematicians.

The reader may wonder, why we did not find a pattern using monochromatic rectangles like the one shown in Figure 3a. Since we are using small shapes, there are more solutions with wave-like colorings than solutions that use straight lines. However, if we increased the edge length of the rectangles to a larger size then we would also be able to find a similar coloring to the one shown in Figure 3a.

\section{$5.3 \quad 5$-Coloring}

Figure 8a shows a pattern using a shape similar to a pentagon and with colors appearing on the opposite edge (top/bottom) each time they are used. From this image, we infer that coloring the infinite strip with 5 colors might be done using two rows of pentagons with one edge flat on the edge of the strip and two that connect the edge to the diagonal wave in the center. This pattern is shown in Figure 13. A similar pattern using pentagons was found by Jaan Parts [15] 


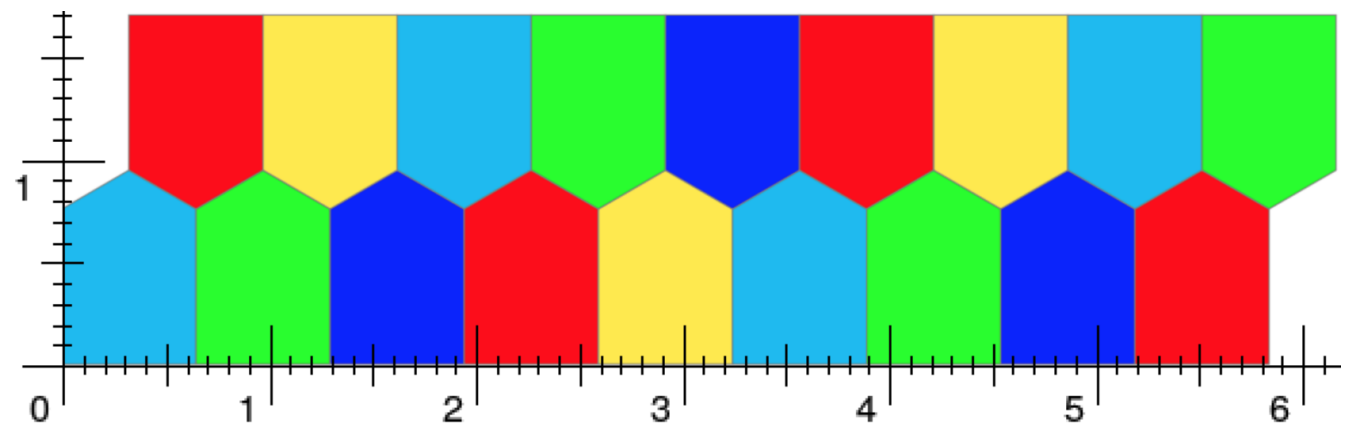

Figure 13: Pentagon tiling of improved height for 5 colors

with a height of 1.625 . This led us to pose the following question.

What is the tallest strip that can be colored with this pattern? To answer this question, we need to make the pentagon as tall as possible, such that for any two points in the pentagon holds that they are less than distance one apart. Let $x$ be the width of the pentagons, and $y$ the length of the vertical edges, and $z$ the height.

We want to find $x, y, z$ that maximizes $y+z$ such that:

- $a^{2}=x^{2}+y^{2} \leq 1$

- $b^{2}=\left(\frac{x}{2}\right)^{2}+z^{2} \leq 1$

- $c^{2}=\left(\frac{3 x}{2}\right)^{2}+(z-y)^{2} \geq 1$

It turns out that there exists a solution for which all three constrains are equal to 1 . In this solution, shown in Figure 14, $x=\frac{\sqrt{3}}{\sqrt{7}}, y=\frac{2}{\sqrt{7}}, z=\frac{5}{2 \sqrt{7}}$. The height of the strip with this pattern is $y+z=\frac{9}{2 \sqrt{7}} \simeq 1.70084$. This is also the optimal solution as the only option to increase $y$ or $z$ is the reduce $x$, but by reducing $x$ the distance of two pentagons with the same color becomes less than 1 .

Validity. To show that this is a valid coloring we need to show that each shape colored with the same color only has points that are more than 1 distance apart. The pattern is repeated infinitely so it suffices to show this for one set. Adjacent like-colored shapes share two sets of parallel line segments we need to consider. These are the diagonals and vertical lines nearest to each other. The distance between parallel lines is the length of the perpendicular line between them. These line segments are offset and there is no perpendicular line intersecting them. Therefore, the closest points between them can be found by finding the closest set of endpoints between them. For both, the closest distance is 1, as shown in Figure 14. Since each shape only has ownership of the points exactly on half of its line segments the distance here is $1+\epsilon$. From here we know all other points are further than 1 because the shape grows further away from these parallel line segments.

\subsection{6-Coloring}

For six colors, we can observe that the pattern found by SArTcolor is similar to the one used to construct a 6 -coloring strip with height 3.668 [2]. This is particularly visible if we compare 


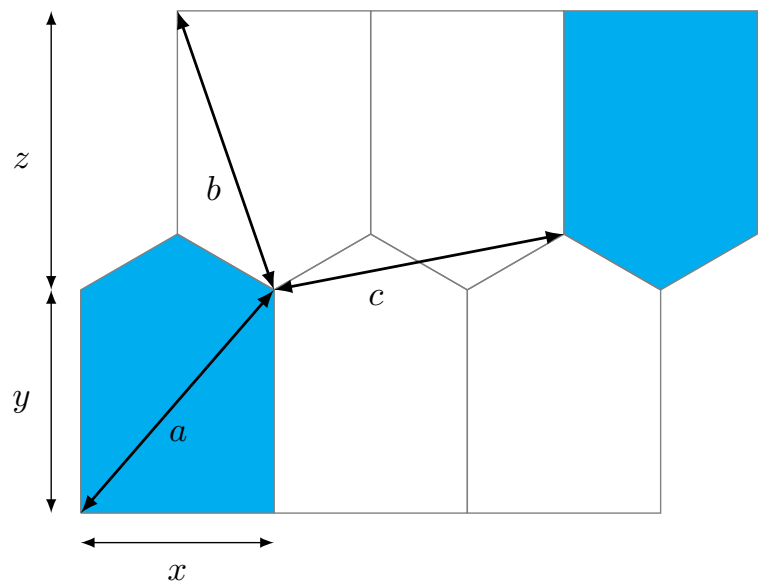

Figure 14: Illustration of the three constraints. The bold distances $a, b$, and $c$ in the shown solution are exactly 1.

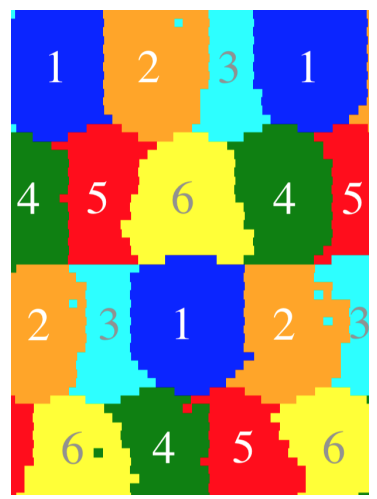

(a) Pattern from the image found by SArTcolor

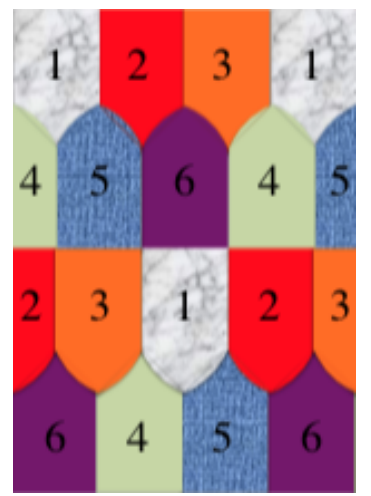

(b) Pattern from known 6-coloring [2]

Figure 15: Comparison between patterns for 6-coloring of strips

the patterns in Figure 3c with Figure 11. To make this comparison more clear, we cropped the pattern from each of the images and plotted them in Figure 15 by assigning a number to each color. Even though the shapes are distorted in the pattern found by SArTcolor because of the scaling factor, we can see that the pattern is the same. Moreover, this pattern was only found when using a scaling factor since it allowed SArTcolor to find a 6-coloring for a height of 3.66. This result supports that SArTcolor can be a useful tool to find these kinds of patterns.

\section{Conclusions and Future Work}

We presented a SAT-based approach to color strips with the unit-distance constraint and to visualize the resulting coloring. Our method can be a useful tool for mathematicians to search for patterns in colored strips or other shapes. Apart from tiling an entire strip, we evaluated the usefulness of scaling: only using the inner part of tiles when computing the unit-distance 
constraint. Strips with larger heights can be colored by scaling and also other patterns emerge. However, we observed cases where the produced colorings are not valid: there exists no coloring for the uncolored outer parts of the tiles.

The emerged patterns are similar to the best known heights for 3 to 6 colors. Our method with and without scaling was able to color a strip with 5 colors with a height larger than the best known bound. We analyzed this pattern and established an improved height of 1.70084.

In future work, we plan to automate the search for a full (unscaled) coloring from a given scaled coloring. This part was done manually in this paper. A possible approach is to use smaller tiles for the uncolored parts. Additionally, we plan to experiment with other shapes, such as triangles. Finally, we would like to improve the quality of the resulting images by applying post-processing techniques to reduce the noise that arises if a tile can have multiple colors.

\section{Acknowledgements}

This work was partially supported by NSF award numbers CCF-1813993, CCF-1762363 and NSF award number ACI-1445606 at the Pittsburgh Supercomputing Center (PSC).

\section{References}

[1] Boris Alexeev. Blog post on polymath16. https://dustingmixon.wordpress.com/2018/06/16/ polymath16-seventh-thread-upper-bounds/\#comment-4827, 2018. [Online; accessed 23-April2020].

[2] Maria Axenovich, JiHyeok Choi, Michelle A. Lastrina, T. McKay, J. Smith, and B. Stanton. On the chromatic number of subsets of the euclidean plane. Graphs and Combinatorics, 30(1):71-81, 2014.

[3] Bruce L Bauslaugh. Tearing a strip off the plane. Journal of Graph Theory, 29(1):17-33, 1998.

[4] Armin Biere, Alessandro Cimatti, Edmund M. Clarke, and Yunshan Zhu. Symbolic model checking without BDDs. In Proceedings of International Conference on Tools and Algorithms for Construction and Analysis of Systems, pages 193-207. Springer-Verlag, 1999.

[5] Armin Biere and Marijn Heule. The Effect of Scrambling CNFs. In Daniel Le Berre and Matti Järvisalo, editors, Proceedings of Pragmatics of SAT 2015 and 2018, volume 59 of EPiC Series in Computing, pages 111-126. EasyChair, 2019.

[6] Felix Bock. Epsilon-colorings of strips. Acta Mathematica Universitatis Comenianae, 88(3), 2019.

[7] James M. Crawford, Ginsberg Matthew L., Eugene M. Luks, and Amitabha Roy. Symmetrybreaking predicates for search problems. In Proceedings of International Conference on Knowledge Representation and Reasoning, pages 148-159. Morgan Kaufmann, 1996.

[8] N. G. de Bruijn and Paul Erdős. A colour problem for infinite graphs and a problem in the theory of relations. Nederl. Akad. Wetensch. Proc. Ser. A, 54:371-373, 1951.

[9] Aubrey D. N. J. de Grey. The chromatic number of the plane is at least 5. Geombinatorics, XXVIII:18-31, 2018.

[10] Allen Van Gelder. Another look at graph coloring via propositional satisfiability. Discrete Applied Mathematic, 156(2):230-243, 2008.

[11] Jun Gu and R. Puri. Asynchronous circuit synthesis with boolean satisfiability. IEEE Transactions on Computer-Aided Design of Integrated Circuits and Systems, 14(8):961-973, Aug 1995.

[12] Marijn J. H. Heule. Trimming graphs using clausal proof optimization. In Thomas Schiex and Simon de Givry, editors, Principles and Practice of Constraint Programming, pages 251-267, Cham, 2019. Springer International Publishing. 
[13] Henry Kautz and Bart Selman. Planning as satisfiability. In Proceedings of European Conference on Artificial Intelligence, page 359-363, USA, 1992. John Wiley \& Sons, Inc.

[14] L. Moser and W. Moser. Solution to problem 10. Can. Math. Bull., 4:187-189, 1961.

[15] Jaan Parts. Blog post on polymath16. https://dustingmixon.wordpress.com/2018/08/28/ polymath16-tenth-thread-open-sat-instances/\#comment-5488, 2018. [Online; accessed 21February-2020].

[16] Jaan Parts. Graph minimization, focusing on the example of 5-chromatic unit-distance graphs in the plane. Geombinatorics, 29, 2020.

[17] Dan Pritikin. All unit-distance graphs of order 6197 are 6-colorable. Journal of Combinatorial Theory, Series B, 73(2):159 - 163, 1998.

[18] Alexander Soifer. The Mathematical Coloring Book. Springer New York, 2009. 